\title{
A NEW METHOD FOR THE EXTRACTION OF DIODE PARAMETERS USING A SINGLE EXPONENTIAL MODEL
}

\author{
S. DIB ${ }^{\mathrm{a}, *}$, M. DE LA BARDONNIE ${ }^{\mathrm{b}}$, A. KHOURY ${ }^{\mathrm{a}}$, \\ F. PELANCHON ${ }^{\mathrm{b}}$ and P. MIALHE \\ ${ }^{a}$ Laboratoire de Physique des Semiconducteurs et Energétique, \\ Faculté des Sciences, Université Libanaise, Fanar. B.P. 90656 Jdeidet, \\ Liban; ${ }^{\mathrm{b}}$ Centre d'Etudes Fondamentales, Université de Perpignan, \\ Avenue de Villeneuve, F-66860 Perpignan, France
}

(Received 23 April 1999; In final form 20 July 1999)

\begin{abstract}
A new method for extracting junction parameters of the single diode model is presented. A least squares method approach considers the deviation $\Delta V=f(I)$ between the experimental current-voltage $(I-V)$ characteristic and a theoretical arbitrary characteristic. A specific case - the $\Delta V$ graph reducing to a straight line - is identified and the knowledge of the slope and of the intercept with the ordinate axis leads to the determination of the junction parameters. The method is applied to the characterization of the emitter-base junction of transistors and the results are discussed.
\end{abstract}

Keywords: Diode; characteristic; junction parameters; linear correlation coefficient

\section{INTRODUCTION}

The $p-n$ junction current voltage $(I-V)$ characteristics have been studied for the past twenty years by many authors. Mathematical models based on the principles of solid-state physics are reasonably well developed. From the one exponential model [1], the $(I-V)$ characteristic

*Corresponding author. 
can be described by the equation:

$$
I=I_{0}\left(\mathrm{e}^{\left(V-R_{S} I\right) / A \beta}-1\right)+\frac{V-R_{S} I}{R_{s h}}
$$

where $\beta=k T / q$ is the thermal voltage, $I_{0}$ is the saturation current, $A$ is the junction ideality factor, $R_{s}$ and $R_{s h}$ are respectively the series and shunt resistances. Equation (1) introduces four parameters $I_{0}, A$, $R_{s}, R_{s h}$, related to internal properties of the electronic device.

Recent studies $[2,3]$ provided discussions to relate these parameters to the space charge recombination processes in $p-n$ junctions. The effect of bulk and surface recombination on the value of the ideality factor of gate controlled diodes has been analysed $[4,5]$. The variation of the junction parameters, with the illumination level, for a solar cell has been studied [6] and associated with the modification of the carrier transport process when illumination increases. The degradation [7] of the junction parameters of transistor devices due to ionizing radiations has been shown to be controlled by the total dose and related to radiationinduced damage. A study of the evolution of the junction parameters [8] of submicronic $n$-MOS transistors following electrical ageing has led to an evaluation of the magnitude of hot carrier degradation and this evolution has been found to be dependent on the device dimensions [9].

Several methods have been proposed to measure the parameters $I_{0}, A, R_{s}, R_{s h}$ by using the description of the experimental junction characteristic obtained with Eq. (1). They were developed for solar cells. Some were based on the consideration of $I-V$ measurements obtained for dark and illumination conditions $/ 2,10-11 /$ or on parameter approximations /12-16/ with numerical process involving determinations around particular points $/ 14,17-19 /$. Others were based on curve fitting methods $/ 6,20 /$ which consider three regions of the characteristic: short circuit current, open circuit voltage and around the maximum power point. All methods require very low level current measurements near the origin used for determination of finite shunt resistance value. Whenever the shunt resistance values is very high, a high quality and sensitive measurement system is necessary to avoid uncertainties in the determination of the parameters.

The aim of this paper is to present a new method to extract the junction parameters from the $(I-V)$ characteristic whenever the shunt 
resistance value is very high. This simple and fast extraction technique involves the high voltage part of the characteristic and can easily be implanted in technological processes for quality control. A simulation is performed to compare the results with theoretical values of the parameters. The method is applied to characterize the base-emitter junction of transistors.

\section{METHOD}

In silicon components, diodes, bipolar transistors and MOSFETs, the junctions are of good quality. For these junctions, the shunt resistance $R_{s h}$ is very high and the expression $\left(V-R_{s} I\right) / R_{s h}$ is small by comparison with the other terms in Eq. (1). Moreover, the calculations are performed here for voltage values greater than $0.4 \mathrm{~V}$ and the term 1 is completely negligible with respect to the exponential term. Taking into account these remarks, relationship (1) is reduced to:

$$
V=R_{s} I+A \beta \ln \frac{I}{I_{0}}
$$

For this method, a similar equation is considered for another characteristic:

$$
V^{\prime}=R_{s}^{\prime} I+A^{\prime} \beta \ln \frac{I}{I_{0}^{\prime}}
$$

where the values of the parameters $R_{s}^{\prime}, A^{\prime}$ and $I_{0}^{\prime}$ are arbitrary. Combining Eqs. (2) and (3), the difference $\Delta V=V^{\prime}-V$ may be written in the form:

$$
\Delta V=\left(R_{s}^{\prime}-R_{s}\right) I+\beta\left(A^{\prime}-A\right) \cdot \ln I+\beta\left(A \cdot \ln I_{0}-A^{\prime} \cdot \ln I_{0}^{\prime}\right) .
$$

When $A=A^{\prime}$, the curve: $\Delta V=f(I)$ becomes a straight line:

$$
\Delta V=\left(R_{s}^{\prime}-R_{s}\right) I+\beta A \cdot \ln \frac{I_{0}}{I_{0}^{\prime}} .
$$

From these results, a numerical process may be elaborated to extract the parameters $R_{s}, A$ and $I_{0}$ of a junction from its experimental $V=f(I)$ characteristic. 
A theoretical characteristic $V^{\prime}=f(I)$ (Eq. (3)) is considered with arbitrary values $R_{s}^{\prime}, A^{\prime}$ and $I_{0}^{\prime}$. Then, $A^{\prime}$ is varied to determine the value $A^{\prime}=A$ so that $\Delta V$ becomes a linear function of the current. It is performed, while varying $A^{\prime}$, by seeking the maximum value of the linear correlation coefficient $R^{2}$ (Fig. 1) between the experimental and the theoretical characteristics.

The slope $a$ and the origin ordinate value $b$ of the obtained straight line in Eq. (5) yield:

$$
R_{s}=R_{s}^{\prime}-a
$$

and

$$
I_{0}=I_{0}^{\prime} \exp \frac{b}{A \beta} .
$$

This procedure leads to obtain, with Eq. (2) a fairly exact fit to the experimental $(I, V)$ characteristic. Figure 2 illustrates this result and shows both an experimental junction $(I, V)$ characteristic (dotted line)

$\Delta \mathrm{V}(\mathrm{V})$

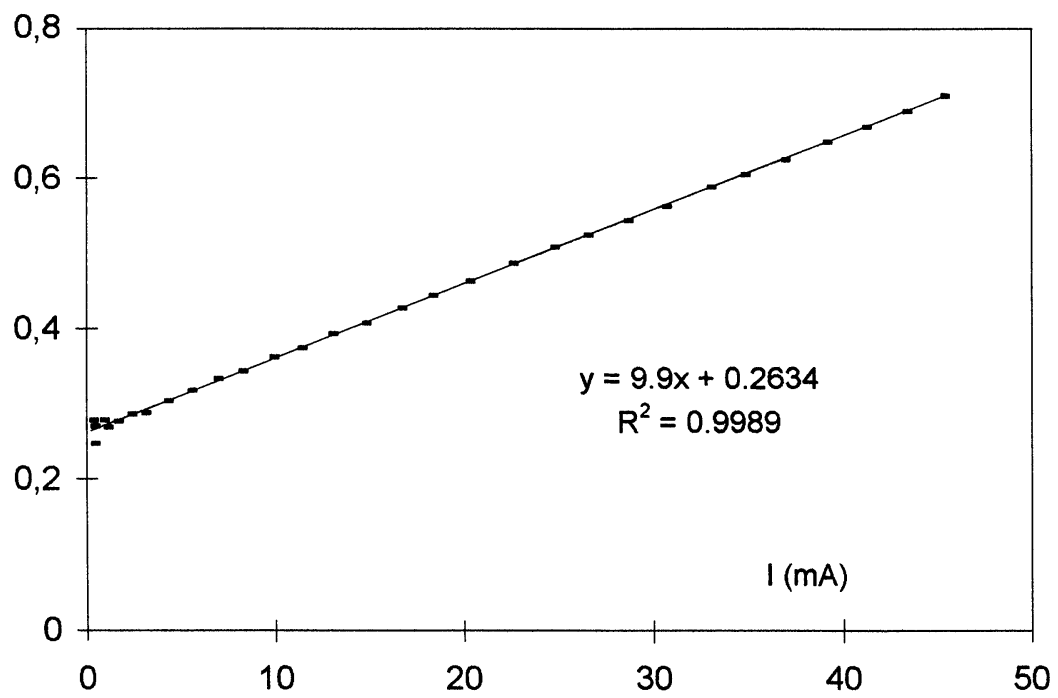

FIGURE 1 Difference $\Delta V$ between an experimental (dotted line) and an arbitrary characteristic versus current $I$ for maximum values of the correlation coefficient ( $A=A^{\prime}=1.89$ ). 


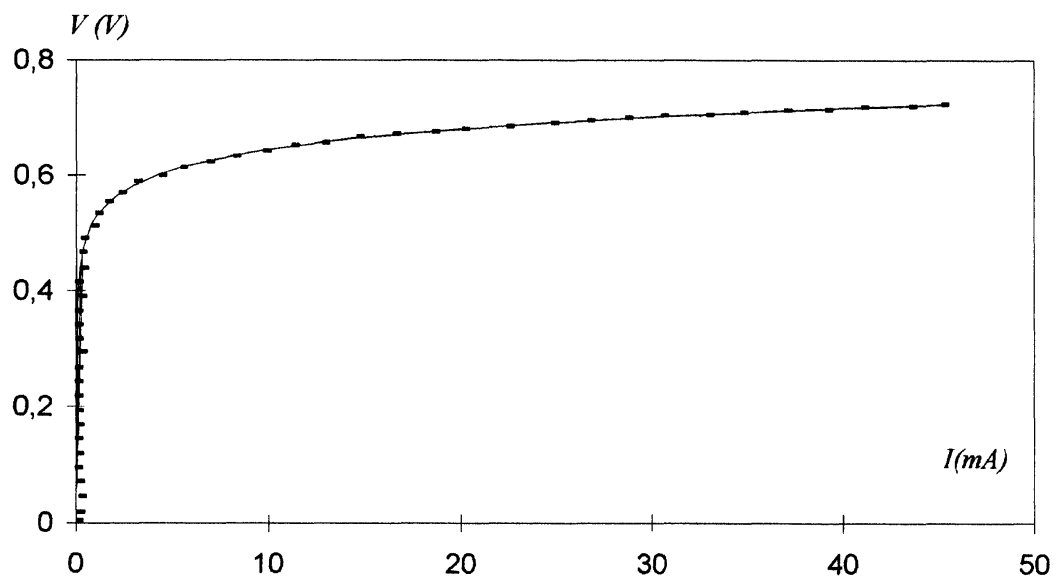

FIGURE 2 Description of the experimental $(I, V)$ characteristic (dotted line) with the one exponential model $\left(A=1.89, R_{s}=0.11 \Omega, I_{0}=2.610^{-8} \mathrm{~A}\right)$.

and the graph of function $V$ given by Eq. (2) for parameters $I_{0}, R_{s}$, and $A$ values extracted by the above procedure.

\section{RESULTS}

Table I shows results obtained with characteristics simulated by using Eq. (1) and a large range of parameter values. $A, R_{s}$ and $I_{0}$ have then been extracted and the ratios of extracted to simulated values have been computed for comparison. For these simulations, each parameter is varied while the others have an arbitrary fixed value. These results point out the accuracy of the method.

Table II shows an application of the method to characterize the base-emitter junction of the transistor $B C 33725$. Twenty measurements of the same experimental junction $(I, V)$ characteristic have

TABLE I Ratios of the extracted to simulated parameter values for simulated characteristics. In each case, only one parameter is varied in a specific variation range, the other are fixed with values $A=1.5, R_{s}=1 \Omega, I_{0}=10^{-8} \mathrm{~A}$

\begin{tabular}{lcc}
\hline & $\begin{array}{c}\text { Simulated parameter: } \\
\text { variation range }\end{array}$ & $\begin{array}{l}\text { Extracted to simulated } \\
\text { parameter value ratio }\end{array}$ \\
\hline$A$ & $1-2$ & $1 \pm 10^{-4}$ \\
$R_{s}(\Omega)$ & $0.25-100$ & $1 \pm 210^{-4}$ \\
$I_{0}(A)$ & $10^{-6}-10^{-13}$ & $1 \pm 610^{-3}$ \\
\hline
\end{tabular}


TABLE II Mean values and standard deviation for parameters extracted from 20 experimental measurements under the same conditions

\begin{tabular}{llll}
\hline & $R_{s}(\Omega)$ & $A$ & $I_{0}(A)$ \\
\hline Mean value & 0.973 & 1.317 & $3.4510^{-12}$ \\
Standard deviation & 0.010 & 0.004 & $0.22810^{-12}$ \\
\hline
\end{tabular}

been performed and registered in separate files. The extraction of the junction parameters from these files allowed to compute the parameter mean values. The very small standard deviation of the values confirms the consistency of the method.

\section{CONCLUSION}

A new method for the extraction of junction parameters is presented. This method is appropriate for the characterization of good quality electronic devices (diodes, transistors, solar cells,...) with a large shunt resistance. It is of practical interest since the fitting of $I-V$ characteristics needs the determination of only one parameter by using a least squares method. The fastness of the procedure makes it easy to implement in industrial process for quality control.

\section{References}

[1] Sze, Physics of Semiconductor Devices, Wiley, New York, 1969.

[2] Charkrabarty, K. and Singh, S. N. (1996). Depletion layer resistance and its effect on $I-V$ characteristics of fully- and partially-illuminated silicon solar cells. SolidState Electronics, 39, 577-581.

[3] Pallarès, J., Marsal, L. F., Correi, X., Calderer, G. J. and Alcubilla, R. (1997) Space Charge recombination in $p-n$ junctions with a discrete and continuous trap distribution. Solid-State Electronics, 41, 17-23.

[4] Chih-Tang Sah (1962). Effects of surface recombination and channel on p-n junction and transistor characteristics. IRE Transactions on Electron Devices, pp. $94-108$.

[5] Kosier, S. L., Wei, A., Schrimpf, R. D., Fleetwood, D. M., Delaus, M. D., Pease R. L. and Combs, W. E. (1995). Physically based comparison of hot-carrierinduced and ionizing-radiation-induced degradation in BJT's. IEEE Transactions on Electron Devices, 42, 436-444.

[6] Charles, P., Mekkoui, I., Bordure, G. and Mialhe, P. (1985). A critical study of the effectiveness of the single and double exponential models for $I-V$ characterisation of solar cells. Solid-State Electronics, 28, 807-820.

[7] de la Bardonnie, M., Maouad, A., Mialhe, P., Elmazria, O., Hoffmann, A., Lepley, B. and Charles, J. P. (1995). Characterization method for ionizing radiation degradation in power MOSFETs. IEEE Transactions on Nuclear Science, 42, 1622-1627. 
[8] de la Bardonnie, M., Mialhe, P. and Charles, J. P. (1998). The evolution of the substrate-drain junction parameters during electrical ageing for $n$-MOS transistor characterization. J. Phys. D: Appl. Phys., 31, 150-156.

[9] de la Bardonnie, M., Mialhe, P., Bendada, E., Blampain, E., Hoffmann, A. and Charles, J. P. (1998). Diode parameter determination applied to LDD-Mosfets for device characterization. Active and Passive Elec. Comp., 20, 157-163.

[10] Veissid, N., Da Cruz, M. T. F. and De Andrade, A. M. (1990). A method for the determination of the standard deviations of the solar cell $I-V$ characteristic parameters. Solar Cells, 28, 351-357.

[11] Chan, D. S. H., Phang, J. C. H. and Wong, H. W. (1987). Experimentally observed deviations from the superposition principle in crystalline silicon solar cells at low illuminations. Solid-State Electronics, 30, 919-925.

[12] Araujo, G. L., Sanchez, E. and Marti, M. (1982). Determination of the two exponential solar cell equation parameters from empirical data. Solar Cells, 5, 199-204.

[13] Polman, A., Van Sark, W. G. J. H. M., Sinke, W. and Saris, F. W. (1986). A new method for the evaluation of solar cell parameters. Solar Cells, 17, 241-251.

[14] Vishnoi, A., Gopal, R., Dwivedi, R. and Srivastava, S. K. (1993). Distributed parameter analysis of dark $I-V$ characteristics of the solar cell: estimation of equivalent lumped series resistance and diode quality factor. IEEE Proceedings, 140, 155-164.

[15] Martil, I. and Gonzalez Diaz, G. (1992). Determination of the dark and illuminated characteristic parameters of a solar cell from $I-V$ characteristics. $J$. Phys., 13, $193-197$.

[16] Sharmar, S. K., Samuel, K. B., Srinivasamurthy, N. and Agrawal, B. L. (1990). Overcoming the problems in determination of solar cell series resistance and diode factor. J. Phys. D. Appl. Phys., 23, 1256-1260.

[17] Kishore, R. (1989). Accurate analytical expressions for the parameters of the single exponential model of the solar cells. Solid-State Electronics, 32, 493-495.

[18] Garrido-Alzar, C. L. (1997). Algorithm for extraction of solar cell parameters from I-V curve using double exponential model. Renewable Energy, 10, 125-128.

[19] Quanxi Jia, Wayne A. Anderson, Enke Liu and Shengliang Zhang (1988). A novel Approach for evaluating the series resistance of solar cells. Solar Cells, 25, 311-318.

[20] Chan, D. S. H., Philips, J. R. and Phang, J. C. H. (1986). A comparative study of extraction methods for solar cell model parameters. Solid-State Electronics, 29, $329-337$. 

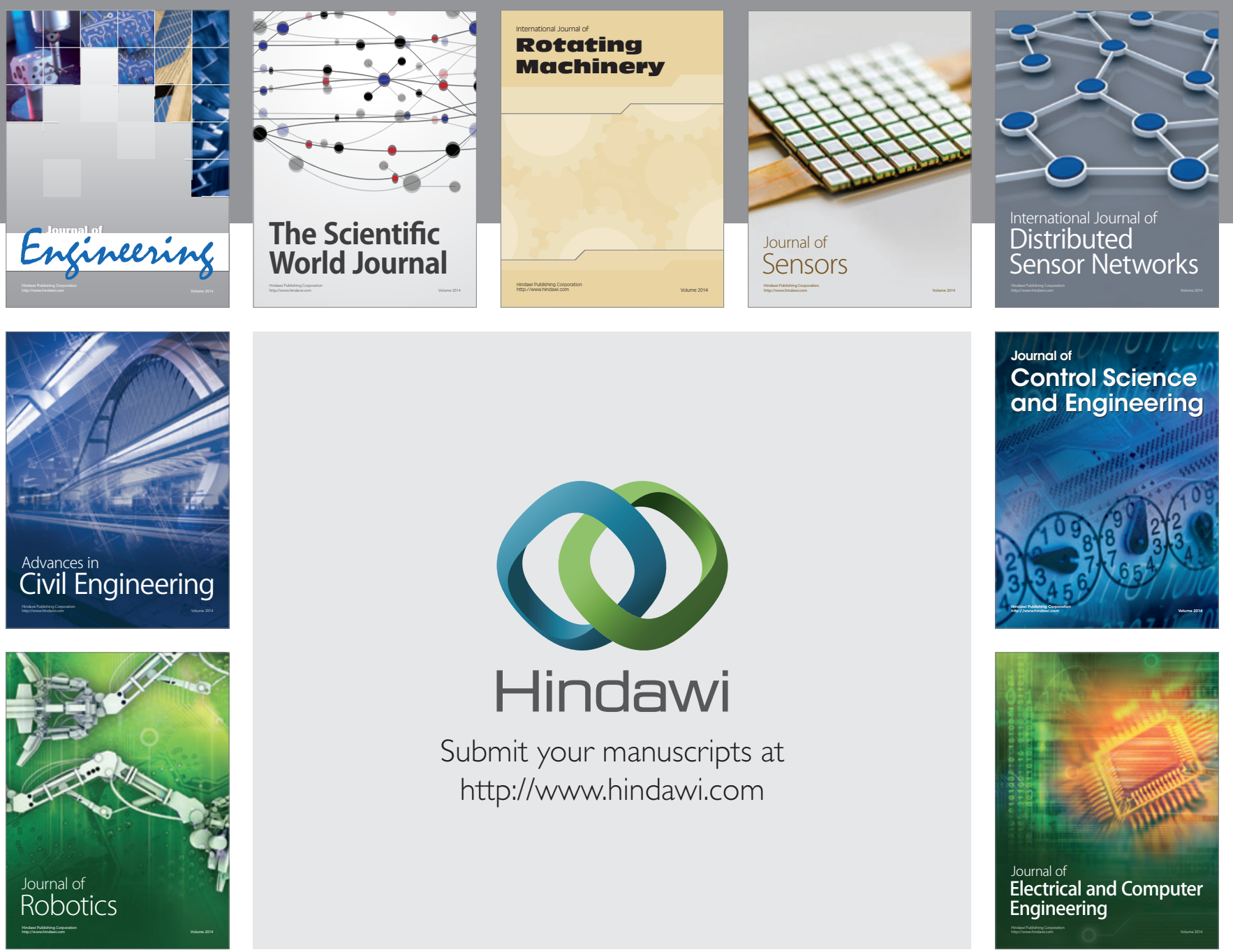

Submit your manuscripts at

http://www.hindawi.com
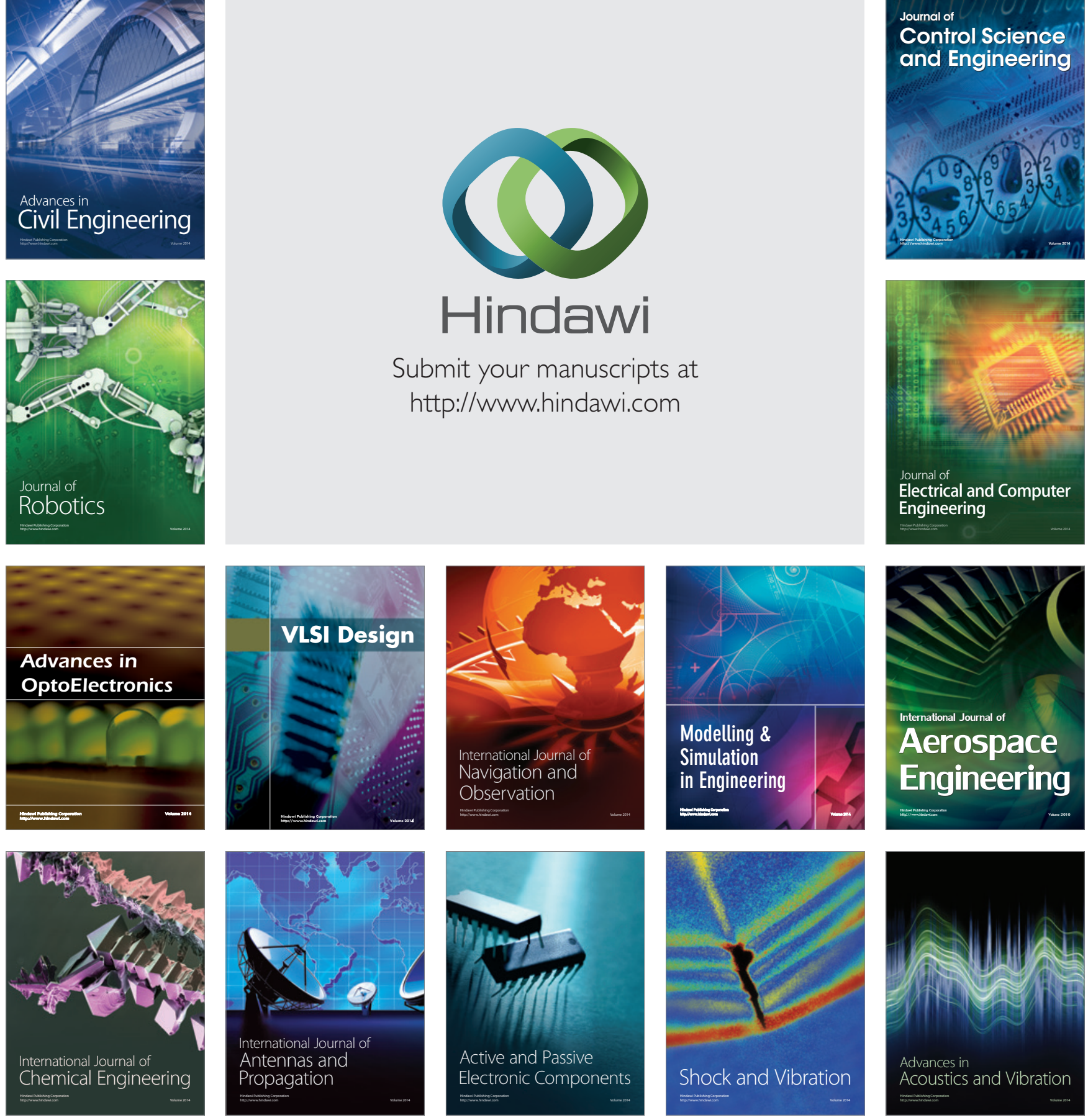